University of Nebraska - Lincoln

DigitalCommons@University of Nebraska - Lincoln

Robert Katz Publications

Research Papers in Physics and Astronomy

October 1959

Two Proposed Experiments for the Detection of the Dirac

Monopole

Robert Katz

University of Nebraska-Lincoln, rkatz2@unl.edu

Darrell R. Parnell

Kansas State University, Manhattan, Kansas

Follow this and additional works at: https://digitalcommons.unl.edu/physicskatz

Part of the Physics Commons

Katz, Robert and Parnell, Darrell R., "Two Proposed Experiments for the Detection of the Dirac Monopole" (1959). Robert Katz Publications. 24.

https://digitalcommons.unl.edu/physicskatz/24

This Article is brought to you for free and open access by the Research Papers in Physics and Astronomy at DigitalCommons@University of Nebraska - Lincoln. It has been accepted for inclusion in Robert Katz Publications by an authorized administrator of DigitalCommons@University of Nebraska - Lincoln. 
Physical Review 116:1 (October 1, 1959), pp. 236-238.

\title{
Two Proposed Experiments for the Detection of the Dirac Monopole
}

\author{
Robert Katz and Darrell R. Parnell * \\ Kansas State University, Manhattan, Kansas \\ *Now at Air Force Cambridge Research Center, Bedford, Massachusetts.
}

Submitted May 8, 1959

A magnetic monopole may be detected by its deflection in an electric field or by the character of the ionization it produces. The electric deflection experiment may be performed in a helium bubble chamber where helical (or spiral) tracks whose axes are parallel to the $D$ lines would be certain evidence for the discovery of the monopole. Previous studies of the ionization have emphasized that the Bragg tail would be missing from a monopole track, as compared to a charged particle. This conclusion must be modified because of the thin-down of tracks of heavy nuclei. The tracks of heavy nuclei thicken up and then thin down as they approach the end of their range. The tracks of monopoles are wedge-shaped, thinning down continuously as they approach the end of their range. Since the track width is due to knockon electrons, or delta rays, any search for the monopole using this criterion must be conducted with electron-sensitive emulsions.

C1959 The American Physical Society. Used by permission.

Online at URL: http://link.aps.org/abstract/PR/v116/p236

DOI: $10.1103 /$ PhysRev.116.236 


\title{
Two Proposed Experiments for the Detection of the Dirac Monopole
}

\author{
Robert Katz and Darrell R. Parnell*
}

Kansas State University, Manhattan, Kansas

(Received May 8, 1959)

\begin{abstract}
A magnetic monopole may be detected by its deflection in an electric field or by the character of the ionization it produces. The electric deflection experiment may be performed in a helium bubble chamber where helical (or spiral) tracks whose axes are parallel to the $D$-lines would be certain evidence for the discovery of the monopole. Previous studies of the ionization have emphasized that the Bragg tail would be missing from a monopole track, as compared to a charged particle. This conclusion must be modified because of the thin-down of tracks of heavy nuclei. The tracks of heavy nuclei thicken up and then thin down as they approach the end of their range. The tracks of monopoles are wedge-shaped, thinning down continuously as they approach the end of their range. Since the track width is due to knock-on electrons, or delta rays, any search for the monopole using this criterion must be conducted with electron-sensitive emulsions.
\end{abstract}

\section{INTRODUCTION}

$\mathbf{I}^{\mathrm{N}}$ NTEREST in the detection of the magnetic monopole is undoubtedly due to the work of Dirac ${ }^{1}$ who related the existence of a quantized electric charge $e$ to a postulated unit pole $g$ by the relationship $g=137 e / 2$. The same result was subsequently derived by Wilson ${ }^{2}$ by quantizing the angular momentum in the electric and magnetic fields of a static assembly of an electron and a magnetic pole. The angular momentum of this field has magnitude $e g / c$ and is directed along the line from a positive charge to a positive pole. Knowledge of the angular momentum in the field of a charge and a pole led Thomson ${ }^{3}$ to derive the magnetic force on a charged particle through a gyroscopic analogy. It is interesting to note in passing that one may construct static classical electromagnetic models possessing the property of spin (identified as the angular momentum in the field) by use of both electric charges and magnetic poles, but not with charges alone nor with poles alone. Here too a particle displaying magnetic moment and spin (such as the neutron) must have an electric moment.

The possibility of binding the monopole to matter was investigated by Malkus, ${ }^{4}$ who concluded that monopoles could be bound to matter with energies comparable to the chemical bond. Thus they might be swept out of matter by strong applied magnetic fields. Goto ${ }^{5}$ has suggested that poles in the cosmic radiation incident on the earth would be trapped in magnetite and that these might be swept into small volumes for detection. The idea of sweeping monopoles from matter has been used by Bradner and Isbell ${ }^{6}$ who attempted to detect monopoles generated within a polyethylene target of the Bevatron by 6.2-Bev protons.

*Now at Air Force Cambridge Research Center, Bedford, Massachusetts.

${ }^{1}$ P. A. M. Dirac, Phys. Rev. 74, 817 (1948) ; Proc. Roy. Soc. (London) A133, 60 (1931).

2 H. A. Wilson, Phys. Rev. 75, 309 (1949).

3 J. J. Thomson, Elements of the Mathematical Theory of Electricity and Magnetism (Cambridge University Press, Cambridge, 1921), fifth edition, p. $396 \mathrm{ff}$.

${ }^{4}$ W. V. R. Malkus, Phys. Rev. 83, 899 (1951)

5 E. Goto, J. Phys. Soc. (Japan) 13, 1413 (1958).

${ }^{6}$ H. Bradner and W. M. Isbell, Phys. Rev. 114, 603 (1959).
Attempts to detect monopoles at the surface of the earth, presumably from cosmic rays, have been reported by Malkus, ${ }^{4}$ who hoped to accelerate monopoles through a solenoid onto track plates, and by Ruark and collaborators, ${ }^{7}$ who searched for appropriate tracks in very clean cloud chambers.

From the failure of these researches to identify a monopole it is evident that the monopole is at best a rare object, and that it will be detected only by clear, easily applied, unequivocal phenomenological criteria. Two such criteria are proposed below.

\section{ELECTROMAGNETIC PROPERTIES OF OF THE MONOPOLE}

It is customary to state in textbooks that "analogous formulas may be derived for magnetic poles" and then to ignore them. As a result the electrical properties of poles are unfamiliar to most physicists. These properties are most easily generated from Maxwell's equations in which magnetic sources are included. ${ }^{8}$ In Gaussian units we write

$$
\begin{array}{ll}
\operatorname{curl} \mathbf{E}=-\frac{1}{c}\left(\frac{\partial \mathbf{B}}{\partial t}+4 \pi \mathbf{J}_{m}\right) ; & \operatorname{curl} \mathbf{H}=-\frac{1}{c}\left(\frac{\partial \mathbf{D}}{\partial t}+4 \pi \mathbf{J}\right) ; \\
\operatorname{div} \mathbf{D}=4 \pi \rho ; & \operatorname{div} \mathbf{B}=4 \pi \rho_{m} .
\end{array}
$$

From these equations it is evident that a current of magnetic poles, or a moving magnetic pole, generates an electric field given by an equation analogous to Ampere's law, but with a left-hand rule corresponding to the minus sign in the equation for curl E. It is evident that all the familiar equations appropriate to charged particles can be transcribed for poles by noting that the set of Maxwell's equations is unchanged if we write $\mathbf{B}$ for $\mathbf{D}, \mathbf{H}$ for $\mathbf{E}, q$ for $q_{m}$, and conversely, and if we replace $t$ by $-t$. In this way we will replace $\mathbf{J}$ by $-\mathbf{J}_{m}, \mathbf{v}$ by $-\mathbf{v}$, and so on. The Lorentz force on a charged particle $\mathbf{F}=q[\mathbf{E}+(\mathbf{v} / c) \times \mathbf{B}]$ may be rewritten for poles as $\mathbf{F}=q_{m}[\mathbf{H}-(\mathbf{v} / c) \times \mathbf{D}]$. Although the latter

\footnotetext{
${ }^{7}$ Fitz, Good, Kassner, and Ruark, Phys. Rev. 111, 1406 (1958).

${ }^{8}$ American Institute of Physics Handbook (McGraw-Hill Book Company, New York, 1957), p. 5-40.
} 
expression is implicit in the relativistic transformations, it has only recently been stated explicitly.9,10 These equations are equally valid for vacuum and for the material medium.

From the Lorentz force on a pole $q_{m}$ we may infer that a magnetic pole will move in a helical path in a uniform electric field. Thus, if a uniform electric field is imposed on any track-producing device and a helical (or spiral) track is detected whose axis is parallel to the $D$ lines, this event may be interpreted as unequivocal evidence for the existence of the monopole. This experiment appears to be feasible in a helium bubble chamber.

In order to evaluate the possibility of performing an electric deflection experiment in a track producing device it is necessary to estimate the magnitude of the field required. Scattering is a major problem so that the detecting medium must be of low $Z$. Since high electric fields are to be impressed on the medium with the possibility of sparking, it is evident that liquid helium has advantages over liquid hydrogen. A compact solid medium which could be sent aloft in rockets might be advantageous, but unfortunately nuclear emulsions are poor insulators.

If we calculate a figure of merit $f$, defined as the ratio of the multiple scattering angle per unit path length to the field deflection angle per unit path length, and compare the behavior of a pole in an electric field to the behavior of a charged particle in a magnetic field, we find

$$
\frac{f_{\text {pole }}}{f_{\text {charge }}}=\frac{B\left(m_{\text {pole }}+M\right)}{D\left(m_{\text {charge }}+M\right)},
$$

where $m$ is the mass of the moving particle and $M$ is the mass of the scattering center. Comparing a pole and charge of equal masses, the electric displacement $D$ which is required to give readable field deflection for a pole is numerically equal to the flux density $B$ which has been found to give satisfactory deflection for the charged particle. This result does not depend on the magnitude of the charge or the pole strength, for both the field deflection and the scattering deflection depend on this magnitude in the same way.

If we suppose the Dirac monopole to have a mass close to that of the deuteron, that a magnetic field of 1000 gauss gives measurable deflection above scattering for the deuteron in the helium bubble chamber, and that the dielectric constant of liquid helium is close to one, we find that an electric field of 1000 statvolts $/ \mathrm{cm}$, or $300 \mathrm{kv} / \mathrm{cm}$, will produce equivalent deflection of the monopole. Such fields are readily attainable, and are in the range of dielectric strengths of commercial dielectrics. By virture of the high ionization potential of helium, the dielectric strength of liquid helium may be

\footnotetext{
${ }^{9}$ H. Semat and R. Katz, Physics (Rinehart and Company, New York, 1958).

${ }^{10}$ E. G. Cullwick, Electromagnetism and Relativity (Longmans, Green, and Company, London, 1957).
}

considerably higher. From the work of Goldburgir on bubble formation in bubble chambers we know that the operation of propane bubble chambers is not adversely affected by electric fields of $15 \mathrm{kv} / \mathrm{cm}$.

In principle the electric deflection experiment is decisive, and is capable of detecting monopoles of any pole strength and any mass. One picture would demonstrate the existence of the monopole and would provide a measurement of $q_{m} / m$. To the best of our knowledge an electric deflection experiment has never before been proposed as a means of detecting the magnetic monopole.

\section{MONOPOLE TRACKS IN NUCLEAR EMULSIONS}

Due to its large pole strength the Dirac monopole is expected to interact strongly with matter, through the influence of the electric field of the moving monopole on atomic electrons. In 1951 this problem was discussed specifically for cloud chambers and for emulsions by Bauer $^{12}$ and by Cole. ${ }^{13}$ Energy loss was found to be almost entirely due to inelastic collision and to bremsstrahlung, as for fast charged particles. Both authors utilized the semiclassical impact parameter method for calculating the loss of energy through ionization. As compared to Bohr's results for charged particles, ${ }^{14}$ the results were those which would be obtained by the substitution of $g v / c$ for $Z e$, for the electric field at a field point due to a pole $g$ moving with velocity $v$ has magnitude $g v / c r^{2}$, while the charged particle generates a Coulomb field $Z e / r^{2}$ under the same conditions. As a result the energy loss due to ionization should be relatively constant down to low velocities, and should fall to zero without a Bragg tail. According to Cole ${ }^{13}$ the distinguishing feature of the track of a monopole is the rapid decrease in ionization toward the end of the track, whereas it is just the opposite for nuclei. But the track of a heavy ion does not achieve a Bragg tail. Instead it displays a characteristic taper or thin-down toward the end of its range. It is necessary to examine the nature of taper tracks in greater detail to determine what distinction remains between the tracks of heavy ions and the tracks of monopoles.

When taper tracks were first observed in cosmic radiation through the use of nuclear emulsions, ${ }^{15}$ the decrease in ionization near the end of the range was interpreted as due to the capture of electrons from the medium when the ion reached the speed of its orbital electrons. Discrepancies in the experimental values of the taper length and the energy at which charge capture was supposed to take place were noted by Lonchamp. ${ }^{16}$ For example, cyclotron accelerated carbon nuclei displayed a thin-down length of $50 \pm 10 \mu$ corresponding

${ }^{11}$ W. I. Goldburg, Phys. Fluids 1, 353 (1958).

12 E. Bauer, Proc. Cambridge Phil. Soc. 47, 777 (1951).

${ }^{13}$ H. J. D. Cole, Proc. Cambridge Phil. Soc. 47, 196 (1951).

${ }^{14}$ N. Bohr, Phil. Mag. 25, 10 (1913); 30, 581 (1915).

${ }_{15}$ Freier, Lofgren, Ney, and Oppenheimer, Phys. Rev. 74, 1818 (1948).

${ }_{16} \mathrm{~J}$. P. Lonchamp, J. phys. radium 14, 433 (1953). 
to an energy of about $50 \mathrm{Mev}$, yet thin-down should not have begun until about $15 \mathrm{Mev}$ for this ion, according to the charge capture theory. Lonchamp ascribed the track width to delta rays. At low ion velocities the delta rays would have insufficient range to broaden a track and the track would appear to thin down. A track would appear to have a solid trunk of silver wherever the delta-ray density exceeded some particular number (say 400 delta rays per 100 microns), depending on the grain size of the emulsion.

Lonchamp was able to reconcile experimental values of the taper length with this theory. Subsequently these conclusions were validated by Skjeggestad ${ }^{17}$ who found experimental values of the track width in the last $150 \mu$ of range in G-5 emulsions in agreement with Lonchamp's theory for nuclei lighter than oxygen. Noting that this theory implies that thin-down is a property of electronsensitive emulsions, Skjeggestad found that the width of $\mathrm{O}^{16}$ tracks is practically independent of length in this region in Ilford $D-1$ emulsions which are insensitive to electrons. We wish to extend Lonchamp's theory of track width to the Dirac monopole.

As given by Mott, ${ }^{18}$ the number of delta rays per unit length of track, $n$, having energy between $W_{0}$ and $W_{1}$, produced in matter having $N$ electrons per unit volume, by an incident particle of charge $Z e$ and velocity $\beta$, is

$$
n=\frac{2 \pi N Z^{2}}{\beta^{2}}\left(\frac{e^{2}}{m c^{2}}\right)^{2}\left(\frac{m c^{2}}{W_{0}}-\frac{m c^{2}}{W_{1}}\right) .
$$

This equation for the delta-ray spectrum may be transcribed to give the delta-ray spectrum produced by a moving monopole of pole strength $g$ by the recipe already given, namely replace $Z$ by $g \beta / e$. Following Lonchamp, we note that a track will display an opaque trunk out to the range of a delta ray of energy $W_{0}$ if there are 400 delta rays per $100 \mu$ of track length having energy between $W_{0}$ and the maximum energy $W_{1}=2 m c^{2} \beta^{2}$ which an electron can acquire in collision with a heavy ion moving with velocity $\beta$. When the equation is solved for $W_{0}$ and numerical values are substituted, we find:

$$
\begin{aligned}
& \text { for charged particles, } W_{0}=\frac{1020}{1.5 \times 10^{5} \beta^{2} / Z^{2}+1 / \beta^{2}} ; \\
& \text { for Dirac monopoles, } W_{0}=\frac{1020}{32+1 / \beta^{2}} .
\end{aligned}
$$

The charged particles display a track of maximum width for $\beta$ approximately equal to $Z^{\frac{1}{2}} / 20$, while the monopole displays no maximum. If we take the mass of a light nucleus as proportional to $2 Z$ we find that the

\footnotetext{
${ }^{17}$ 0. Skjeggestad, Nuovo cimento 8, 927 (1958).

${ }_{18}$ N. F. Mott, Proc, Roy, Soc. (London) 124, 425 (1929).
}

energy at which the track width is maximum is proportional to $Z^{2}$. These equations are plotted as functions of $\beta$ for the monopole and for $Z=10,20,30$, and 40 in Fig. 1, where an auxiliary scale has been provided to indicate the width of the track in $G-5$ emulsion, from electron range-energy relations given by Voyvodic. ${ }^{19}$

We conclude that electron sensitive emulsions are good detectors for Dirac monopoles. Although the exact thickness of the monopole track depends on the particular emulsion and processing, the sample data used here yield a monopole track width which increases regularly from its ending and reaches a value of about $15 \mu$ at $\beta=\frac{1}{2}$, in $G-5$ emulsion. A quick calculation shows that a nucleus of charge 25 will display a maximum thickness of about $17 \mu$ at $\beta=\frac{1}{4}$, or at about $1.5 \mathrm{Bev}$ under the same conditions. The width of this track will diminish to about $6 \mu$ at $\beta=\frac{1}{2}$. According to Demers, ${ }^{20}$

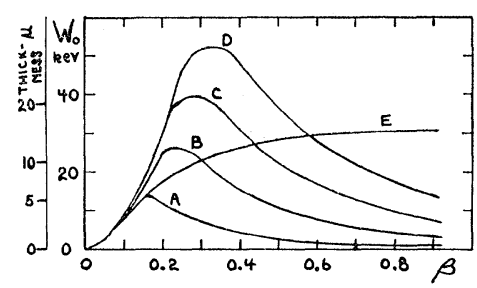

FIG. 1. The energy $W_{0}$ of delta rays at which the delta-ray density is just sufficient to block transmitted light through the tracks of nuclei of charge $10(A), 20(B), 30(C), 40(D)$, and the tracks of Dirac monopoles $(E)$, as a function of $\beta=v / c$. An auxiliary scale indicates the track width in microns in $G-5$ emulsion.

these energies correspond to residual ranges of $400 \mu$ and $4 \mathrm{~mm}$, respectively, in $G-5$ emulsion. We can therefore state a clear phenomenological criterion for scanners of electron-sensitive emulsions: Look for wedge-shaped tracks whose trunk achieves a thickness of about $15 \mu$ and which are at least $500 \mu$ long. Such a track may be the Dirac monopole.

We have scanned five 2-in. $\times 4$-in. Ilford $G-5$ emulsions exposed at $100000 \mathrm{ft}$ for monopole tracks according to these characteristics. In these pellicles 29 tracks suitable for measurement were found and these all displayed characteristics consistent with their identification as heavy ions.

\section{ACKNOWLEDGMENTS}

We wish to thank R. D. Hill, G. Ascoli, J. Weneser, and J. V. Lepore for their comments on various aspects of this problem; H. Bradner for sending us his results prior to publication; and D. Haskin for the plates used in this investigation. A grant from the Research Corporation has helped to support this research.

${ }^{19} \mathrm{~L}$. Voyvodic, Progress in Cosmic-Ray Physics (Interscience Publishers, Inc., New York, 1953), Vol. 2, p. 261.

${ }^{20} \mathrm{P}$. Demers, Ionographie (Les Presses Universitaires de Montreal, Montreal, 1958), p. 252 ff. 